\title{
A critical review of citrulline malate supplementation and exercise performance
}

\author{
Lewis A. Gough ${ }^{1}$ (ए) S. Andy Sparks ${ }^{2} \cdot$ Lars R. McNaughton $^{2} \cdot$ Matthew F. Higgins ${ }^{3} \cdot$ Josh W. Newbury ${ }^{1}$ Eric Trexler ${ }^{4}$. \\ Mark A. Faghy ${ }^{5}$. Craig A. Bridge ${ }^{2}$
}

Received: 1 April 2021 / Accepted: 20 July 2021 / Published online: 21 August 2021

(c) The Author(s) 2021

\begin{abstract}
As a nitric oxide (NO) enhancer, citrulline malate $(\mathrm{CM})$ has recently been touted as a potential ergogenic aid to both resistance and high-intensity exercise performance, as well as the recovery of muscular performance. The mechanism has been associated with enhanced blood flow to active musculature, however, it might be more far-reaching as either ammonia homeostasis could be improved, or ATP production could be increased via greater availability of malate. Moreover, CM might improve muscle recovery via increased nutrient delivery and/or removal of waste products. To date, a single acute $8 \mathrm{~g}$ dose of CM on either resistance exercise performance or cycling has been the most common approach, which has produced equivocal results. This makes the effectiveness of CM to improve exercise performance difficult to determine. Reasons for the disparity in conclusions seem to be due to methodological discrepancies such as the testing protocols and the associated test-retest reliability, dosing strategy (i.e., amount and timing), and the recent discovery of quality control issues with some manufacturers stated (i.e., citrulline:malate ratios). Further exploration of the optimal dose is therefore required including quantification of the bioavailability of NO, citrulline, and malate following ingestion of a range of CM doses. Similarly, further well-controlled studies using highly repeatable exercise protocols with a large aerobic component are required to assess the mechanisms associated with this supplement appropriately. Until such studies are completed, the efficacy of CM supplementation to improve exercise performance remains ambiguous.
\end{abstract}

Keywords Metabolism $\cdot$ High-intensity exercise $\cdot$ Supplements $\cdot$ Resistance training $\cdot$ Nitric oxide

$\begin{array}{ll}\text { Abbreviations } \\ \text { CM } & \text { Citrulline malate } \\ \text { NO } & \text { Nitric oxide } \\ \text { ATP } & \text { Adenosine triphosphate } \\ \text { MAS } & \text { Malate aspartate shuttle }\end{array}$

Communicated by Michael Lindinger.

Lewis A. Gough

lewis.gough@bcu.ac.uk

1 Research Centre for Life and Sport Science (CLaSS), Human Performance and Health Research Group, Birmingham City University, Birmingham, UK

2 Sports Nutrition and Performance Research Group, Department of Sport and Physical Activity, Edge Hill University, Ormskirk, UK

3 University of Derby, Derby, UK

4 Stronger By Science LLC, Raleigh, NC, USA

5 Human Sciences Research Centre, University of Derby, Derby, UK

$\begin{array}{ll}\text { MDH } & \text { Malate dehydrogenase } \\ \text { NIRS } & \text { Near infrared spectroscopy } \\ \text { PFK } & \text { Phosphofructokinase } \\ \text { TCA } & \text { Tricarboxylic acid } \\ \text { HR } & \text { Heart rate } \\ \text { GI } & \text { Gastrointestinal } \\ \text { GVT } & \text { German volume training } \\ \text { VO }_{2 \text { peak }} & \text { Peak oxygen consumption } \\ \text { EMG } & \text { Electromyography }\end{array}$

\section{Introduction}

Considerable research attention has recently been placed on the physiological signalling molecule, nitric oxide (NO) (Jones et al. 2020). Augmenting NO synthesis through exogenous substances may improve skeletal muscle function and performance through improved blood flow, contractility, and mitochondrial respiration (Stamler and Meissner 2001). Typical strategies to increase 
NO activity include the ingestion of green leafy vegetables and/or beetroot juice and L-citrulline (Jones 2020). Indeed, L-citrulline is known to exert positive effects on exercise performance and recovery (Gonzalez and Trexler 2020). More recently, however, a direct NO precursor called citrulline malate (CM) has been touted to have ergogenic potential, which is the combination of L-citrulline and malate (Gonzalez and Trexler 2020). The mechanisms of $\mathrm{CM}$ might be more far-reaching as a result, due to the synergistic impact of both components (i.e., L-citrulline and malate) at the intramuscular level (Wax et al. 2015). Specifically, malate has been suggested to increase the rate of ATP production by mitigating lactate production during states of high flux; and by doing so allowing for continued pyruvate and energy production (Wax et al. 2016). Furthermore, the malate-aspartate shuttle (MAS) may be more efficient following CM ingestion, thereby improving ATP availability (Wu et al. 2007; Agudelo et al. 2019). Based on these promising findings and additional mechanisms compared to L-citrulline supplementation alone, it is plausible to suggest CM supplementation could be a worthwhile ergogenic aid.

Since the early work of Bendahan et al. (2002), research has primarily focused on the potential ergogenic effects of $\mathrm{CM}$ supplementation on resistance exercise performance. In an early study, a greater contribution of oxidative ATP synthesis (34\% increase) to energy production was observed with chronic ingestion of CM for 15 days ( $6 \mathrm{~g}$ day) (Brendahan et al. 2002). This study, however, focused on sedentary individuals who complained of fatigue and included no placebo condition and therefore, the application to athletes is limited. Nonetheless, recent work has addressed these limitations and investigated acute doses of CM against a placebo and the associated effects on short-term exercise that encompasses a large anaerobic component in trained and untrained individuals. To date, equivocal responses to $\mathrm{CM}$ supplementation have been reported, which makes the performance-enhancing potential ambiguous. Moreover, CM ingestion could improve acute recovery from exercise due to the augmentation of blood flow and the indirect increase in nutrient delivery and clearance of waste metabolites (Wax et al. 2015; Glenn et al. 2017). This could have important implications for athletes who may have minimal recovery from competition/training due to the high frequency of exercise bouts in their schedules (e.g., team sports, track and field). This review therefore builds upon a previous review by Gonzalez and Trexler (2020) who recently discussed the efficacy $\mathrm{CM}$ ingestion to improve exercise performance within a larger, more general review of NO enhancing supplements, by offering a more in-depth discussion on the potential mechanisms associated with CM supplementation. This is followed by a discussion of findings to date in respect of improving exercise performance and/or recovery, including modifying factors such as the exercise type and duration, ingestion strategy (dose and timing), and the safety of CM supplementation.

\section{Proposed ergogenic mechanisms}

The ingestion of CM was originally prescribed to enhance the muscle performance of patients suffering from asthenia and to facilitate the recovery of muscle function resulting from acute diseases (Brendahan et al. 2002). As an organic salt, $\mathrm{CM}$ and is formed through the combination of L-citrulline $\left(\mathrm{C}_{6} \mathrm{H}_{13} \mathrm{~N}_{3} \mathrm{O}_{3}\right)$, a non-essential amino acid involved in the urea cycle, and malate (or malic acid, $\mathrm{C}_{4} \mathrm{H}_{6} \mathrm{O}_{5}$ ), a tricarboxylic acid (TCA) intermediate (Bendahan et al. 2002; da Silva et al. 2017; Glenn et al. 2017; Wax et al. 2015). Outside of the common practice of oral supplementation of beetroot juice, L-citrulline ingestion has been the most researched nutritional strategy to stimulate NO production (Gonzalez and Trexler, 2020). This is likely explained by studies that show L-citrulline ingestion to be the most efficient means of elevating plasma arginine concentrations, which in turn, produces NO (Schwedhelm et al. 2008).

The proposed mechanism for CM ingestion is firstly dependent on the citrulline component via the L-arginineNO pathway, such that following NO synthesis the smooth muscle may relax leading to vasodilation (Vanhoutte et al. 2016; Fig. 1). In turn, these vasodilatory properties may improve the delivery of blood (and oxygen) to and from the active musculature during exercise (Wax et al. 2015). Trexler and colleagues (2019a, 2019b) have recently questioned this mechanism, however, using the near-infrared spectroscopy (NIRS) technique to quantify blood flow. Indeed, Trexler et al. (2019a) reported $8 \mathrm{~g} \mathrm{CM}$ ingestion $2 \mathrm{~h}$ prior to exercise had no effect on muscle blood flow (CM: $3.78 \pm 0.26$, placebo: $3.72 \pm 0.26 \mathrm{ml} . \mathrm{min}^{-1} .100 \mathrm{ml}^{-1}$ ) or oxygen consumption (CM: $1.15 \pm 0.11$, placebo: $1.16 \pm 0.11 \mathrm{mLO}_{2}$. $\min ^{-1} .100 \mathrm{~g}^{-1}$ ) during $3 \mathrm{~min}$ of leg extension exercise (one rep every $4 \mathrm{~s}$ ). These findings were replicated during maximal effort leg extension exercise consisting of 5 sets of 30 maximal-effort concentric leg extensions interspersed with $1 \mathrm{~min}$ of passive rest between sets (Trexler et al. 2019b). The authors findings were likely due to the small rises in NO observed in these studies, as they were not significantly different to the placebo $(\mathrm{CM}: 15.3 \pm 1.1$; placebo: $13.4 \pm 1.1 \mu \mathrm{mol} \cdot \mathrm{L}$; Trexler et al. 2019b). Alternatively, the failure to select a dynamic whole-body exercise might have led to no improvement, as this might not have sufficiently stressed the aerobic mechanisms associate with ingestion of this supplement. Finally, whilst the use of NIRS is widely considered a reliable technique (Lucero et al. 2018), it is unclear if this technique is sensitive enough to detect changes following supplement intake. The evidence to date 
Fig. 1 A schematic representation of the mechanisms associated with citrulline malate supplementation. Bold denotes the active ingredient. Left: NO-derived mechanism, Right: Ammonia clearance mechanism. *Denotes evidence is either speculative or has only been observed in mice. (Schematic created in BioRender.com)

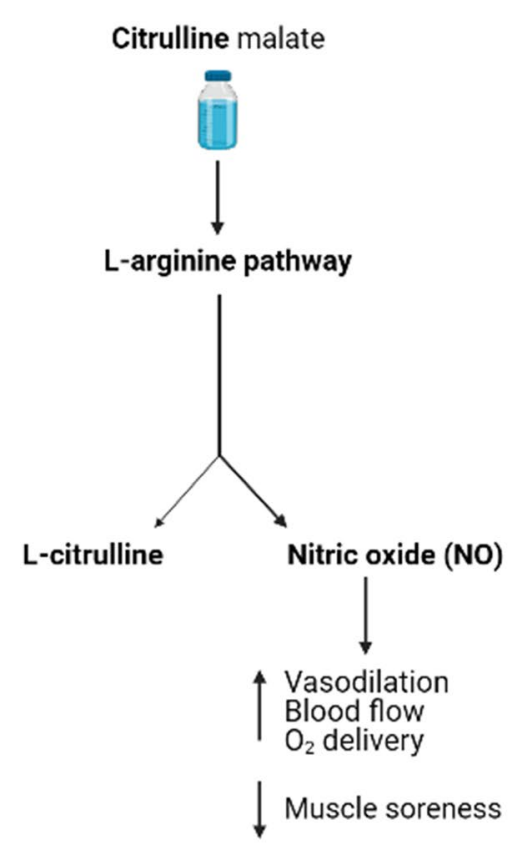

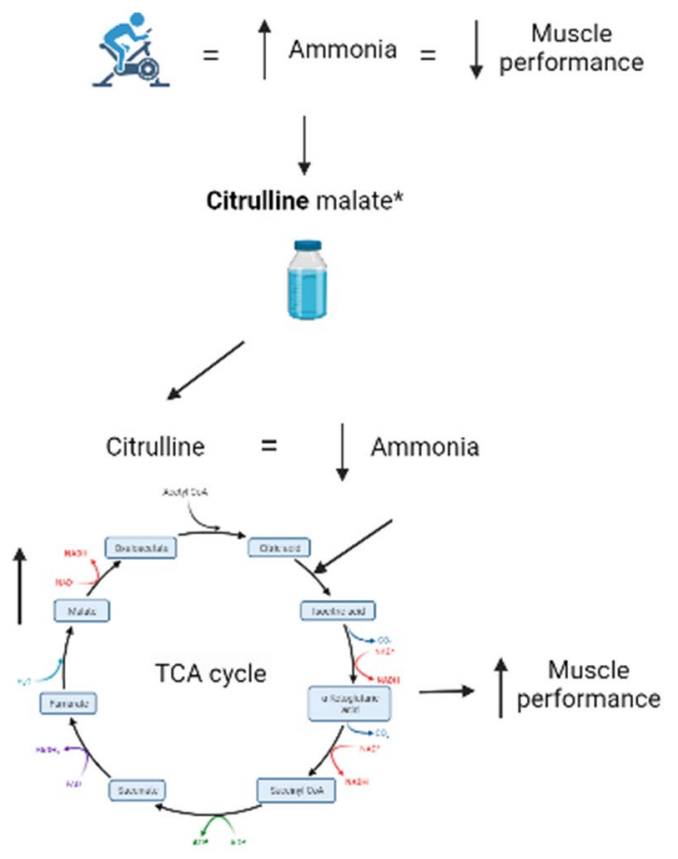

specifically on CM supplementation suggests that enhanced blood flow caused by the citrulline component is not the acting mechanism, although further experimental research is required.

An alternative mechanism could be from the citrulline component of CM, as this may assist with ammonia elimination during the urea cycle (Bendahan et al. 2002; Fig. 1). This is important due to the increased ammonia production observed during high-intensity exercise, and the association of these changes with muscle fatigue (Gonzalez and Trexler 2020). Specifically, high ammonia concentrations facilitate the production of lactate during anaerobic glycolysis by activating phosphofructokinase (PFK). In turn, this prevents oxidative metabolism of pyruvate to acetyl-CoA and hinders the ATP supply to skeletal muscle (Hargreaves and Spriet 2020). Citrulline may exert its ergogenic effects through this mechanism by detoxifying ammonia during high-intensity exercise, thereby enhancing the aerobic utilization of pyruvate and ATP supply to skeletal muscle (Gonazalez and Trexler, 2020). Indeed, Takeda et al. (2011) reported ingestion of citrulline $\left(250 \mathrm{mg} \cdot \mathrm{kg}^{-1} \mathrm{BM}\right)$ reduced ammonia accumulation by $90.1 \mu \mathrm{g} / \mathrm{dL}^{-1}$ (citrulline: $351.3 \pm 35.3$ vs. control: $441.4 \pm 61.3 \mu \mathrm{g} . \mathrm{dL}^{-1}$ ) and increased time to exhaustion swimming by approximately 9 min in mice $($ citrulline $=24 \mathrm{~min}$, placebo $=15 \mathrm{~min}$ ). Importantly, post-exercise lactate concentrations were lower for the citrulline-supplemented group, which in turn, supports the mechanism of enhancing aerobic utilization during exercise. This has not been replicated in humans, however, and as such, it remains to be confirmed if this mechanism occurs during human exercise.
Greater ergogenic benefits may be possible in addition to the L-citrulline mechanisms with the addition of malate (Fig. 2). The primary role of malate is to function as a tricarboxylic acid cycle intermediate, which may play an important role in the rate of ATP production (Brendahan et al. 2002). One of the most critical controls of the rate of aerobic ATP production is oxaloacetate, and as malate is dehydrogenated into this compound in the TCA cycle, it may offer an explanation to the purported additive effects of CM over L-citrulline alone (Brendahan et al. 2002). Equally, malate is suggested to play a critical role (amongst four other TCA intermediates) in ancillary reactions that can alter the concentrations of TCA intermediaries and result in positively affecting the fluxes in and out of the TCA cycle (Gibala et al. 2000). Whilst these mechanisms sound promising, no study to date involving CM supplementation and exercise performance has provided evidence of these mechanisms occurring. Admittedly, this is difficult given the complexities and lack of measurement techniques, and most evidence supporting this theory to date is based on mathematical approaches/ modelling. It is not possible therefore to confirm at this time whether they are important during exercise. Nonetheless, the synergistic mechanisms of L-citrulline and malate (i.e., CM) do offer hope that these mechanisms could synergistically improve exercise performance. Future research should directly compare L-citrulline vs. CM ingestion to assess if the addition of malate does offer additive benefits to exercise performance.

A contemporary mechanism may be either through the increase in gene expression, or increased efficiency of the MAS, which is caused by elevations in PGC- $1 \alpha$ in 


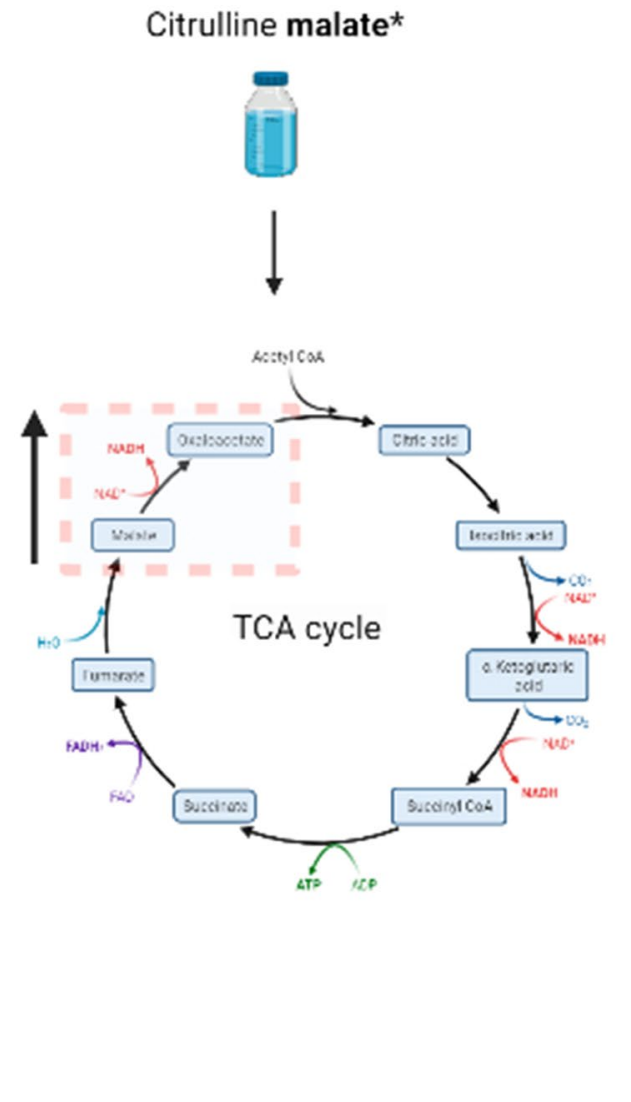

Fig. 2 A schematic representation of the mechanisms associated with citrulline malate supplementation. Bold denotes the active ingredient. Left: Increased bioavailability of malate mechanism, Right: Increased

exercising musculature. These physiological changes can elevate aspartate and glutamate levels and increase the expression of glycolysis and MAS genes (Wu et al. 2007; Agudelo et al. 2019). As a result, increases in the transfer of fuel-derived electrons to mitochondrial respiration may occur, which in theory, should improve energy utilisation (Wu et al. 2007; Fig. 2). Indeed, evidence in mice has shown that supplementation of L-malate increased the activity of malate dehydrogenase (MDH) in a dose-response manner, as higher doses reported more activity (Control mice: $15.7 \pm 2.4$ vs. 0.2 g. $\mathrm{kg}^{-1} \mathrm{BM} \cdot \mathrm{day}^{-1}$ dose: $24.3 \pm 3.5$ vs. $0.6 \mathrm{~g}$. $\mathrm{kg}^{-1} \mathrm{BM} \cdot \mathrm{day}^{-1}$ dose: $26.4 \pm 3.7$ ) (Wu et al. 2007). Mechanistically, as MDH is a rate-limiting enzyme for MAS, an increase in activity could suggest an increase in efficiency and therefore ATP supply. Similarly, MDH yields oxaloacetate by dehydrogenating L-malate to increase overall rates of TCA flux and MAS. Support for these mechanisms can be found in Wu et al. (2007), such that time to exhaustion swimming was improved in both the $0.2 \mathrm{~g} \cdot \mathrm{kg}^{-1} \mathrm{BM} \cdot \mathrm{day}^{-1} \mathrm{~g}$ $(+26.1 \% ; 620 \pm 141 \mathrm{~s})$ group and $0.6 \mathrm{~g} \cdot \mathrm{kg}^{-1} \mathrm{BM}^{\mathrm{day}}{ }^{-1}$ $(+28.5 \% ; 631 \pm 134 \mathrm{~s})$ versus the control $(491 \pm 145 \mathrm{~s})$. However, no data is available from using human participants

\section{Citrulline malate*}

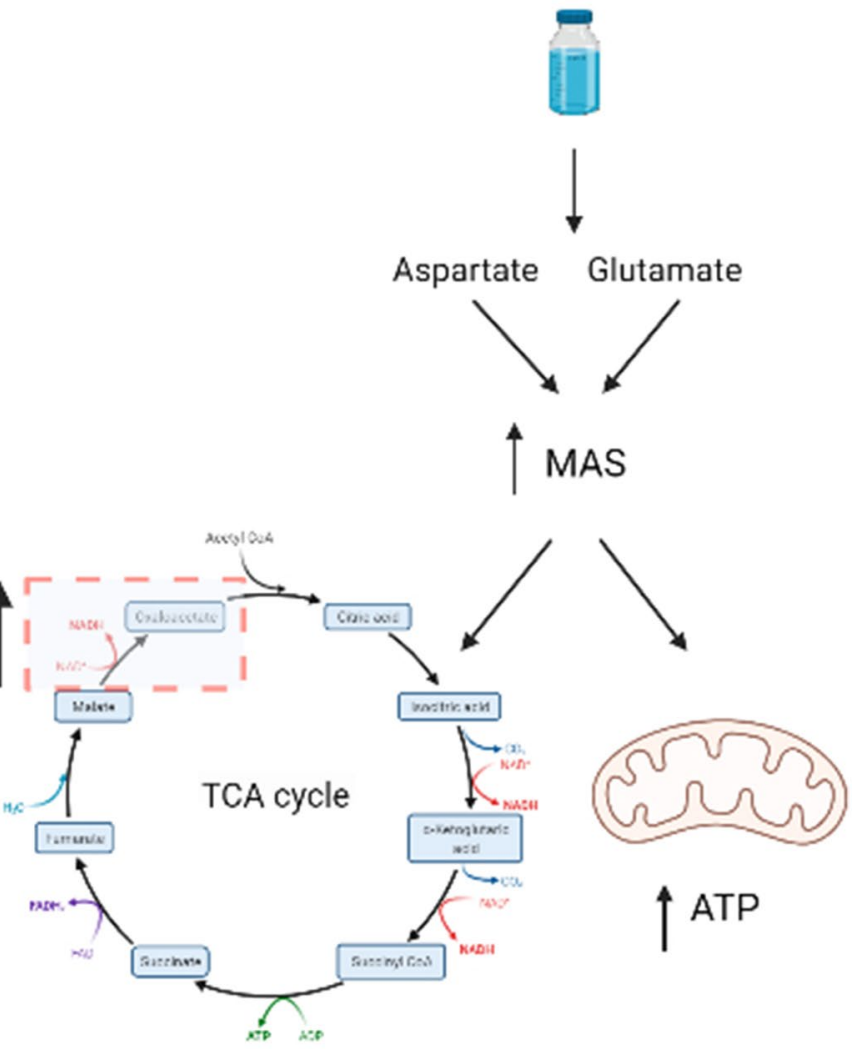

efficiency of Malate Aspartate Shuttle (MAS). *Denotes evidence is either speculative or has only been observed in mice. (Schematic created in BioRender.com)

to corroborate these findings in mice, whilst Wu et al. (2007) also chronically supplemented L-malate, not CM. Further research in humans is therefore required to confirm this mechanism.

Supplementation of CM is suggested to reduce muscle soreness from exercise, via the purported increased blood flow mechanism (Wax et al. 2015; da Silva et al. 2017). This could be important for either subsequent performance when recovery between bouts is limited (i.e., track and field) and/ or overall quality and quantity of chronic training. In a large study of forty-one men, ingestion of $8 \mathrm{~g} \mathrm{CM} 1 \mathrm{~h}$ before a resistance training bout led to decreases in muscle soreness at both $24 \mathrm{~h}(-40 \%)$ and $48 \mathrm{~h}(-41 \%)$, respectively, compared to a placebo (Perez-Guisado et al. 2010). However, there was no subsequent performance bout to substantiate whether the reduction in soreness translates to improved performance. In a more thorough study (da Silva et al. 2017), CM supplementation ( $6 \mathrm{~g}, 1 \mathrm{~h}$ before exercise) failed to improve muscle soreness when more reporting points $(24,48$, and $72 \mathrm{~h})$ were used compared to Perez-Guisado et al. (2010). Importantly, da Silva et al. (2017) also reported no improvement in subsequent performance for neither leg press exercise, nor hack 
squat to exhaustion. Whilst the lack of differences observed could be attributed to the low $6 \mathrm{~g}$ dose adopted by da Silva et al. (2017), a more recent study has corroborated these findings using an $8 \mathrm{~g}$ dose, $1 \mathrm{~h}$ before exercise (Chappel et al. 2018). Based on the current evidence that CM supplementation does not enhance blood flow and thereby increase nutrient delivery and/or remove waste metabolites, this might explain why no positive impact has been reported to date for muscle soreness and subsequent exercise performance.

\section{Dose, timing, and safety}

The most commonly employed dose of CM is a single acute $8 \mathrm{~g}$ dose (Gonzalez and Trexler 2020), which appears to reflect early work observing performance benefits during resistance exercise using this dose (Perez-Guisado et al. 2010; Table 1). However, an earlier dose-response study (Moinard et al. 2008) investigated the pharmacokinetics of $2,5,10$, and $15 \mathrm{~g}$ of citrulline within eight healthy volunteers and demonstrated that larger doses could be more appropriate. The authors reported that peak citrulline concentration occurred with a $15 \mathrm{~g}$ dose $\left(3849 \pm 190 \mu \mathrm{mol}^{-1} \mathrm{l}^{-1}\right)$, which was significantly higher $(+28.4 \%)$ compared to $10 \mathrm{~g}$ citrulline $\left(2756 \pm 170 \mu \mathrm{mol} . \mathrm{l}^{-1}\right)$. It is plausible such larger doses (i.e., $>10 \mathrm{~g}$ ) will increase the likelihood of securing an ergogenic benefit during exercise, which questions the use of a $8 \mathrm{~g}$ dose in most research to date. It is worth noting, however, that Moinard et al. (2008) only used citrulline, rather than the combined intake of CM. The extent to which the malate may influence the peak concentration and pharmacokinetics is therefore currently unknown and warrants further investigation. Indeed, to achieve an intake of $10 \mathrm{~g}$ of L-citrulline, ingestion of around 3-5 kg of fresh watermelon (highest concentration of all foods) would be required and this is not only impractical for athletes (Davis et al. 2011), but would also not contain a sufficient dose of the malate component. Given the large dose required for any possible ergogenic effect, supplementation is only practical via ingestion of marketed sports supplements or the raw chemical compound.

There appears to be limited diversity in the timing of $\mathrm{CM}$ ingestion with most studies opting for 1-h ingestion before exercise (Gonzalez and Trexler 2020). This approach seems to be informed by previous research reporting ergogenic effects using similar dosing strategies (Wax et al. 2015, 2016; Farney et al. 2019), rather than the time to reach peak concentrations of citrulline (Moinard et al. 2008; Cunniffe et al. 2016). Few studies investigating exercise performance have included concomitant measurements of peak plasma citrulline, which is likely due to the cost of such procedures. Indeed, Cunniffe et al. (2016) reported that following $12 \mathrm{~g}$ of CM plasma citrulline concentration was $343 \pm 41 \mu \mathrm{M}$, compared to $39 \pm 12 \mu \mathrm{M}$ following the placebo. A similar magnitude of change was also observed for plasma ornithine concentration $(9.5 \pm 3.1 \mu \mathrm{M}$ vs. $2.4 \pm 1.6 \mu \mathrm{M})$. However, the time-course changes in these markers cannot be determined, as only a single blood sample was taken at $60 \mathrm{~min}$. Equally, the 12-g dose ingested was higher than that typically used in $\mathrm{CM}$ research, therefore the changes in plasma citrulline concentration following the most common $8 \mathrm{~g}$ dose are unknown. Nonetheless, a study by Moinard et al. (2008) reported that following a range of doses of citrulline $(2,5$, 10 , and $15 \mathrm{~g}$ ), peak concentration of citrulline occurred at approximately $1 \mathrm{~h}$, however, this rapidly declined 15-30 min after the initial peak regardless of the dose ingested. Both plasma arginine and ornithine also displayed a similar pattern of peak and clearance. Further research investigating time-course changes in citrulline concentration following $\mathrm{CM}$ supplementation is required to determine the optimal strategy, although the best recommendation at the moment would be $1 \mathrm{~h}$ prior to exercise. It can also be concluded that the 'ergogenic window' is likely to be small and therefore determination of the peak absorption characteristics are likely to be important to securing an ergogenic benefit.

Most research investigating CM ingestion and exercise performance reports that a 2:1 ratio of citrulline:malate has been used. Recent research has challenged these reports, however, such that many $\mathrm{CM}$ manufacturers failed to reach the purported ratios (Chappel et al. 2018). Importantly, the ratios reported in a study using nuclear magnetic resonance spectroscopy reported most nutritional companies/suppliers only provided a CM ratio of approximately $1.6: 1$, with some as low as 1.1:1 (Chappel et al. 2018). Based on ingesting $8 \mathrm{~g}$ of $\mathrm{CM}$ with a 1.1:1 ratio, individuals are only ingesting $4.2 \mathrm{~g}$ instead of $5.3 \mathrm{~g}$ (the latter based on a 2:1 ratio). These potential quality control issues have ramifications for the existing body of work, future research, and its use by athletes in practice. Based on multiple studies using citrulline in isolation and reporting ergogenic effects (Gonzalez and Trexler 2020), it is likely the citrulline component of CM is vital to secure ergogenic benefits. It is plausible as a result that the equivocal findings in respect of $\mathrm{CM}$ ingestion and exercise performance could also be partly attributed to the amount of ingested citrulline being lower than intended. Researchers should therefore consider an independent assessment of supplement quality and authenticity to be able to gain assurances the dose ingested is as intended. If an independent assessment is not available, based on the findings from Chappel et al. (2018) only Trade Ingredients (www.trade ingredients.com) were close to the required ratio, and therefore this is the best-known source at this time.

The effects of citrulline supplementation on biomarkers associated with health outcomes have been previously investigated; however, limited evidence on CM exists. Indeed, Moinard et al. (2008) reported that ingestion of a 


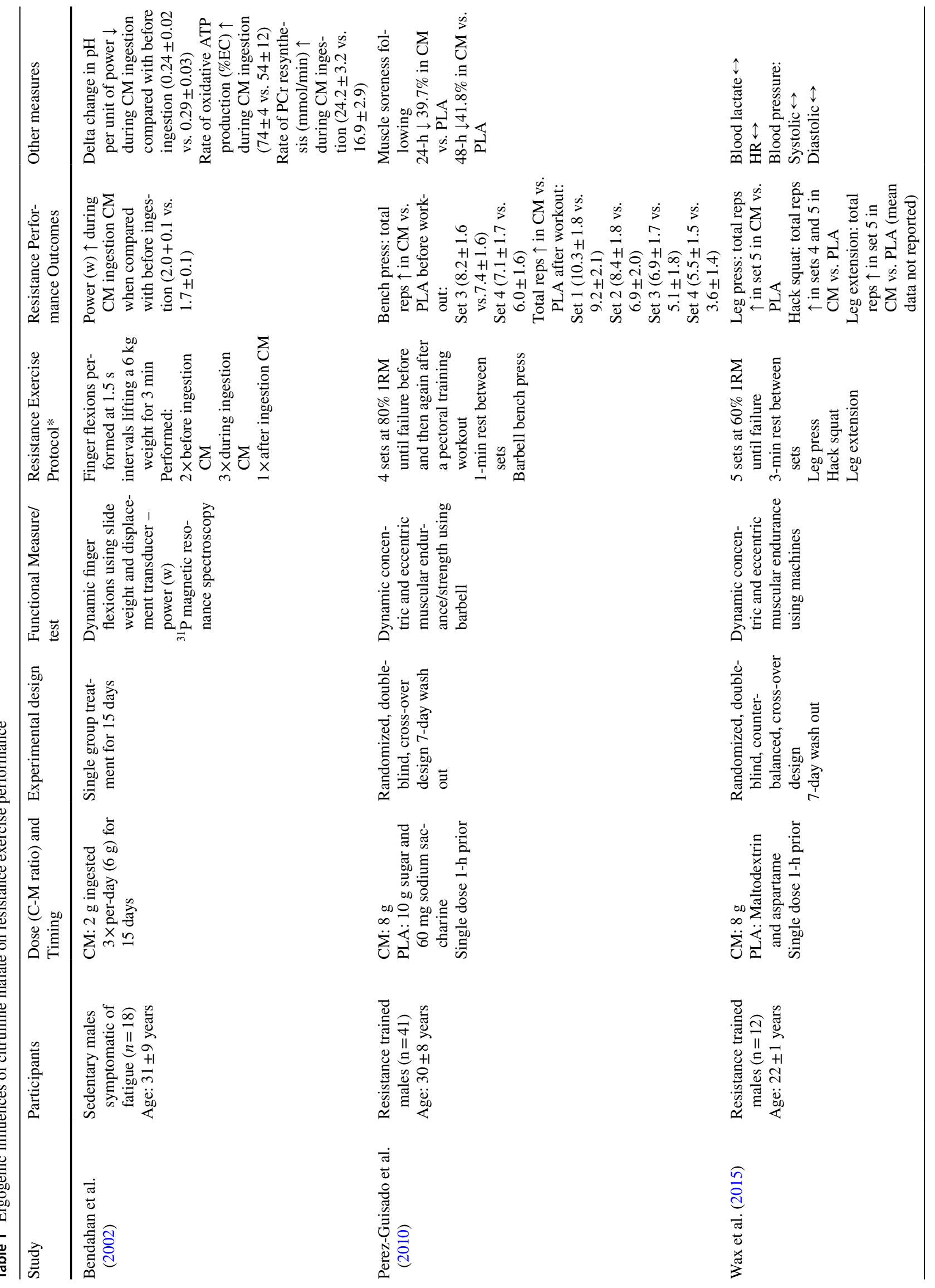




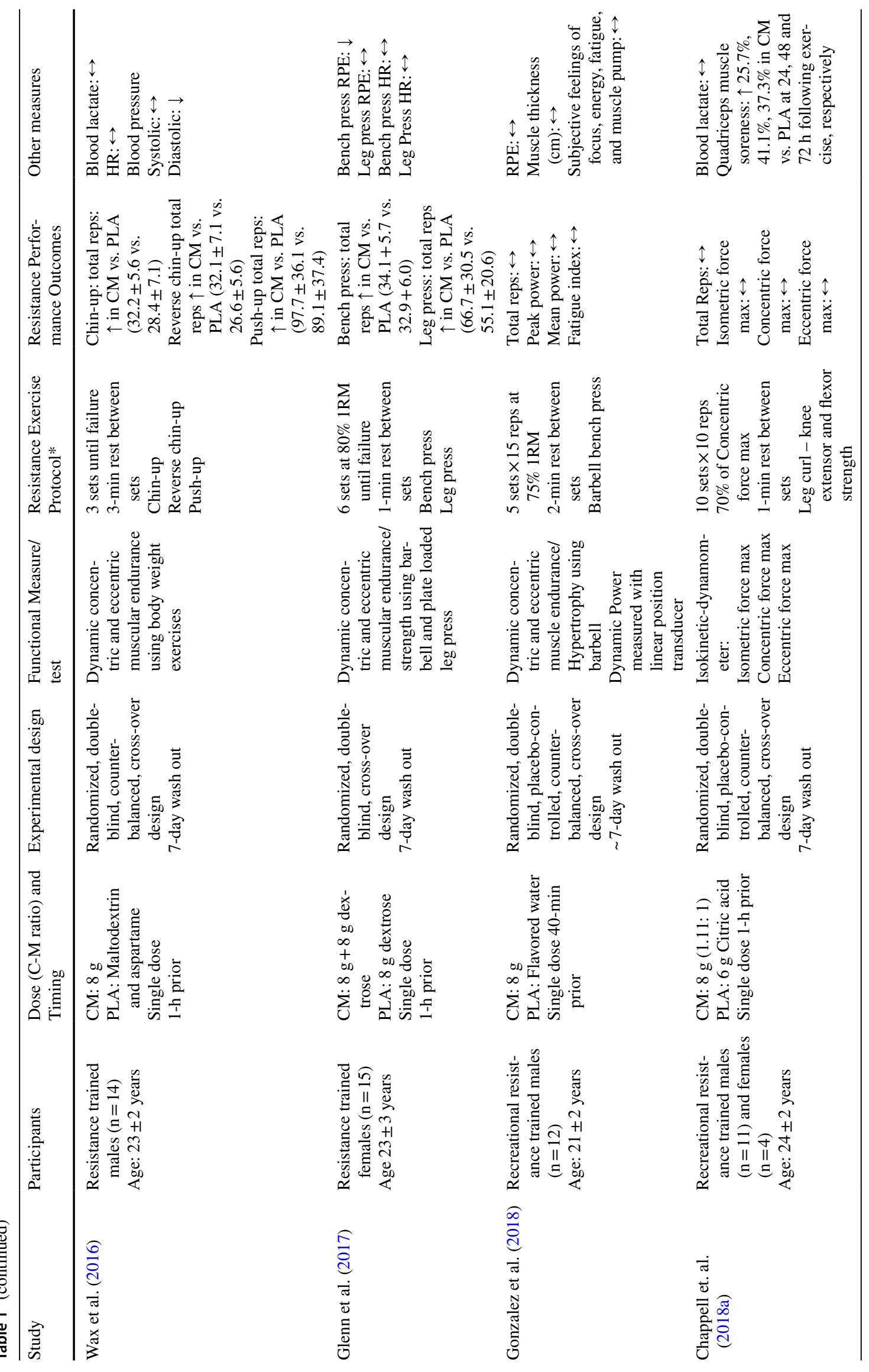




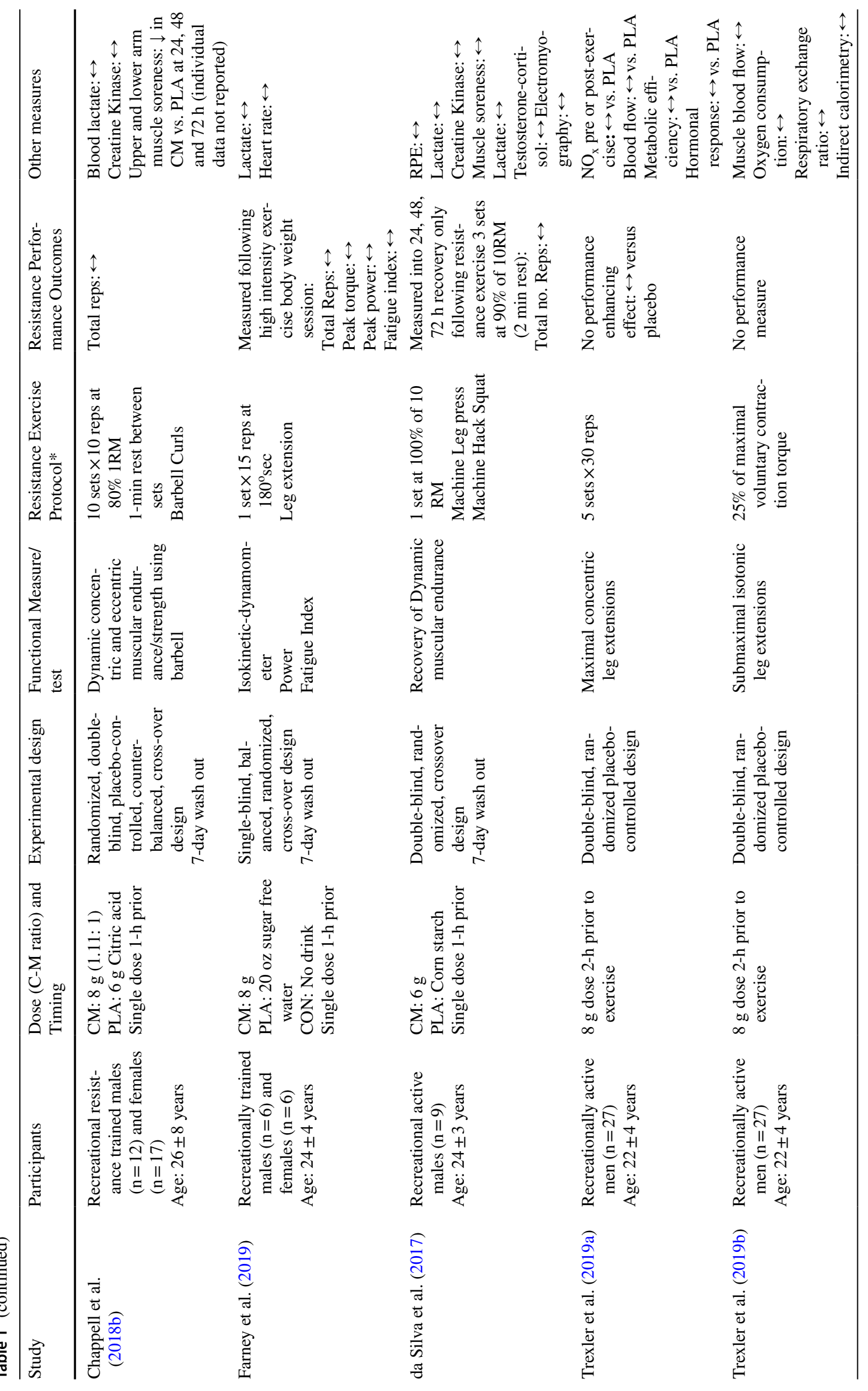




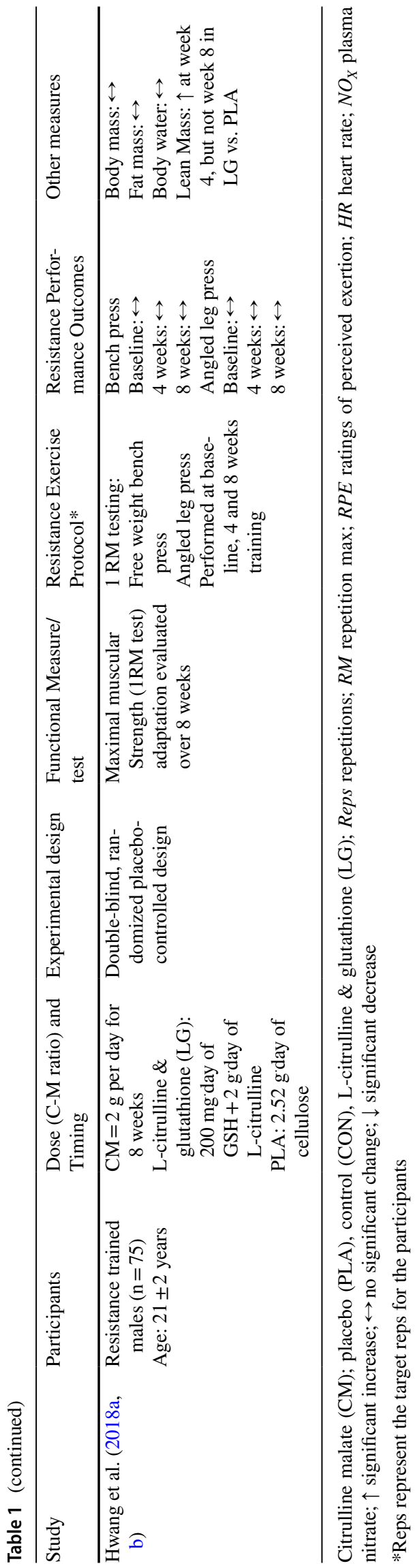

range of doses between $2-15 \mathrm{~g}$ of citrulline had no adverse effect on hematological markers (leucocytes, polymorphonuclears, lymphocytes, monocytes, erythrocytes, $\mathrm{Hb}$ ) or biochemical markers (calcium, total proteins, albumin, C-reactive protein, urea, creatinine, glucose, cholesterol, triacylglycerol). Unfortunately, no study as comprehensive as the Moinard et al. (2008) study exists for CM supplementation, although a small amount of research has studied health outcomes alongside supplementation (Brendahan et al. 2002; Casonatto et al. 2019). Indeed, Brendahan et al. (2002) supplemented CM chronically at $6 \mathrm{~g} \mathrm{day}^{-1}$ for 15 days, in a group of 18 sedentary males and reported no negative experiences. No objective health markers were measured, however, and the reports were subjective views from the authors. Casonatto et al. (2019), however, reported that following supplementation of $6 \mathrm{~g}$ of $\mathrm{CM}$, both diastolic and systolic blood pressure was reduced over a 24 -h period following exercise (40-min run/walk at $60-70 \%$ HR reserve) within a group of 40 hypertensive individuals. The applications of these findings would be difficult to apply to athletes, as they are likely to undertake more routine ingestion of CM compared to hypertensive patients. Considering the studies to date have only used an acute dose, and not used trained athletes, the safety of longer-term supplementation of CM requires further investigation. It is worth noting, however, that generally the ingestion of nitrate-based supplements are considered safe for consumption (Sindelar et al. 2012).

A common issue with ingestion of amino acids is the onset of gastrointestinal (GI) discomfort, whereby vomiting and diarrhea have been reported following ingestion of related amino acids such as arginine and ornithine in doses of between 6 and $12 \mathrm{~g}$ (Grimble 2007). Notably, the side effects reported with CM supplementation seem to be less severe. Indeed, Glenn et al. (2017) reported no differences in GI discomfort between CM supplementation and placebo following acute ingestion of $8 \mathrm{~g}$ within resistance-trained females, whilst larger doses of $12 \mathrm{~g}$ also seemed to be well-tolerated within a group of trained male cyclists (Cunniffe et al. 2016). These differences could be related to different mechanisms of citrulline uptake, such that it can be taken up from the lumen by multiple transport systems, including $\mathrm{B}^{0,+}$ (Bahri et al. 2008). In comparison, arginine and ornithine are rapidly saturated within the intestine and can therefore induce osmotic diarrhea. Moreover, these factors could explain why citrulline seems to have increased bioavailability compared to similar compounds, such as arginine (Breuillard et al. 2015; Moinard et al. 2008) and why larger doses can be tolerated. The findings for $\mathrm{CM}$ supplementation are therefore promising for real-world application in a performance setting, as no study to date has reported any significant GI discomfort following a range of doses. 


\section{Effects of citrulline malate on exercise performance}

Initial investigations have demonstrated that a single acute $8 \mathrm{~g}$ dose of $\mathrm{CM}$ ingested $1 \mathrm{~h}$ before exercise can enhance dynamic muscular endurance and strength performances, within resistance-trained males and females (Gonzalez and Trexler, 2020; Table 1). Perez-Guisado et al. (2010) used 41 resistance-trained males to perform four sets of barbell bench press at $80 \%$ 1RM (1-min rest between sets) following ingestion of $8 \mathrm{~g} \mathrm{CM}, 1-\mathrm{h}$ prior to exercise. Repetitions to failure in all but the first two sets of bench press before the workout were improved following CM ingestion. Performance responses have been replicated in resistancetrained females whilst performing six sets of bench press and plate-loaded leg press exercise at 80\% 1RM (1-min rest between sets) using a similar dosing strategy (Glenn et al. 2017). Furthermore, in a series of investigations by Wax and colleagues $(2015,2016)$, ingestion of $8 \mathrm{~g}$ of CM 1-h before exercise increased the repetitions to failure during leg press and hack squat at 60\% 1RM, and in a series of bodyweight exercises (chin-ups, push-ups), respectively. These changes were only subtle across these studies when expressed as an effect size (range: 0.23-0.59), which could suggest that CM's ergogenic action might be more modest in such settings. Nonetheless, these small improvements across multiple sessions might improve training adaptation across a training block.

In contrast to the preliminary investigations assessing the ergogenic effects of CM supplementation on resistance exercise, no benefit to German Volume Training (GVT) protocols has been reported (Chappell et al. 2018a, $2018 \mathrm{~b}$ ). The ingestion of $8 \mathrm{~g} \mathrm{CM} 1 \mathrm{~h}$ before exercise did not influence the total number of repetitions to failure during barbell curls $(10 \times 10$ repetitions at $80 \% 1 \mathrm{RM})$ (Chappell et al. 2018a) or isokinetic dynamometer leg curls $(10 \times 10$ repetitions at $70 \%$ of concentric maximum force, 1-min rest between sets) (Chappell et al. 2018b). The latter protocol also showed no change in maximal isometric, concentric and eccentric force using this dosing strategy. Mitigating factors to explain the lack of performance improvement could be either the use of a CM compound disproportionate to the target ratio of citrulline and malate, or the reliability of the exercise protocol. Specifically, both studies by Chappell and colleagues $(2018 \mathrm{a}$, b) reported that the citrulline:malate ratio in the $\mathrm{CM}$ administered to participants was much lower than purported by the manufacturer (2:1 vs. 1.1:1). This equates to only around half of the stated dose of citrulline, which in turn, may have reduced the ergogenic potential. Lastly, GVT resistance training protocols do not seem to have any established test-retest reliability to date, which makes it difficult to determine small but meaningful changes in performance.

Some studies also confer no ergogenic effects of $\mathrm{CM}$ ingestion on dynamic or isokinetic muscular power indices (Farney et al. 2019; Gonzalez et al. 2018; Trexler et al. 2019a, 2019b). Indeed, Gonzalez et al. (2018) used a linear position transducer to examine the influence of $8 \mathrm{~g} \mathrm{CM}$ (40 min before exercise) on power indices whilst performing 5 sets of up to 15 repetitions of barbell bench press exercise at 75\% 1RM (2-min rest between sets). The authors reported no effect on peak power, mean power, fatigue index, or total repetitions performed by the group of resistance-trained males $(n=12)$. These findings agree with arguably the most comprehensive study to date by Trexler et al. (2019a) reporting ingestion of $8 \mathrm{~g} \mathrm{CM} 2 \mathrm{~h}$ before exercise had no impact on peak torque, average torque, or total work during 5 sets of 30 maximal effort, concentric leg extensions $\left(180^{\circ} \cdot \mathrm{s} ; 1\right.$-min rest between sets) compared to a placebo. The authors also reported no mechanistic evidence to suggest $\mathrm{CM}$ improves blood flow, metabolic efficiency, or lactate clearance. These findings have been corroborated during submaximal isotonic concentric leg extensions at $25 \%$ of maximal voluntary contraction torque (Trexler et al. 2019b). A caveat to both Gonzalez et al. (2018) and Trexler et al. (2019a; b) might be the ingestion timing of $\mathrm{CM}$ however, as both were outside of the 1-h before exercise timing used in studies reporting a positive effect on performance or where data suggests peak $\mathrm{NO}$ occurs (Moinard et al. 2008). Furthermore, it is unknown if the mechanisms of action would have been observed during full-body dynamic exercise where there is a higher aerobic and metabolic demand, as CM ingestion seems to be more appropriate for this type of exercise (see proposed ergogenic mechanisms section).

Positive effects of CM ingestion on cycling performance are scarce, with only three studies to date employing a randomised, placebo-controlled crossover study design (Glenn et al. 2017; Cunniffe et al. 2016; Gills et al. 2021). Indeed, Glenn et al. (2017) showed that $8 \mathrm{~g}$ of CM 1-h before exercise failed to improve mean power output or fatigue index during a cycling Wingate, within a group of 17 trained tennis players. Similarly, Cunniffe et al. (2016) observed no improvement during $10 \times 15 \mathrm{~s}$ cycling sprints ( $30 \mathrm{~s}$ active recovery) despite the use of a higher $12 \mathrm{~g}$ dose of CM 1-h before exercise. The short-duration exercise protocols in these studies do not investigate the more aerobically derived mechanisms associated with CM supplementation, however, and this could explain the lack of ergogenic benefit. Nonetheless, in a contemporary study (Gills et al. 2021) employing a time to volitional tolerance $\left(\mathrm{T}_{\mathrm{LIM}}\right)$ protocol at a much lower exercise intensity $\left(90 \% \mathrm{VO}_{2 \text { peak }}\right)$, no improvement in cycling capacity was observed between ingestion of $8 \mathrm{~g} \mathrm{CM}$ versus a placebo, within a group of 28 males $(\mathrm{p}=0.94$; PLA: $315.4 \pm 137.7 \mathrm{~s} ;$ CM: $314.1 \pm 107.1 \mathrm{~s})$. 
It is plausible that the lack of ergogenic effects could be attributed to the trained participant cohort in the cycling studies $\left(\mathrm{VO}_{\text {2peak }}\right.$ range $\left.=56.3-58.1 \mathrm{ml} \cdot \mathrm{kg}^{-1} \cdot \mathrm{min}^{-1}\right)$, such that a recent point-counterpoint debate suggests due to an elevated NO at baseline compared to their untrained counterparts this could blunt the ergogenic effect (Hultström et al. 2015). Alternatively, the variation that is typically seen during $\mathrm{T}_{\text {LIM }}$ tests could explain the null effect, as these protocols require a large difference in performance to reach statistical significance compared to either fixed duration or distance time trials (Currell and Jeukendrup 2008). Considering these studies utilised the same dose and timing of ingestion as other studies demonstrating ergogenic effects in resistance exercise performance, the use of CM seems ineffective at improving cycling performance in trained individuals. Nonetheless, future research should combine CM ingestion and a time trial cycling bout with a predominantly aerobic demand to ascertain if a competitive edge could be gained.

Few studies have considered the ergogenic potential of CM supplementation in supporting the recovery of muscle function (da Silva et al. 2017) and/or adaptive properties of musculature in humans (Hwang et al. 2018a, b) (Table 1). Specifically, chronic supplementation of either $2 \mathrm{~g}$ of CM, $2 \mathrm{~g} \mathrm{~L}$-citrulline, and $200 \mathrm{mg}$ glutathione, or a placebo ( $2.52 \mathrm{~g}$ cellulose) per day over 8 weeks did not affect maximal muscular strength development (via bench press and leg press exercises) (Hwang et al. 2018a, b). Interestingly, whilst no significant difference was found, a large effect size (Hedges $g=1.8$ ) was reported for reductions in fat mass at 8 weeks. A possible explanation for the null effect on performance and/or adaptation may be that the CM dose was too low, as this was significantly reduced compared to the dose typically used to produce ergogenic effects ( $2 \mathrm{~g}$ vs $8 \mathrm{~g}$ ). However, this dose may be sufficient to help with a reduction in fat mass and therefore support overall athlete body composition. With this being the only study to date investigating chronic ingestion of $\mathrm{CM}$, more research is required using higher doses that might subsequently improve the probability of securing any potential benefit to exercise performance and/or body composition.

A study investigating acute recovery from exercise has displayed null effects of CM supplementation (da Silva et al. 2017). The authors reported ingestion of $6 \mathrm{~g}$ of $\mathrm{CM}$ 1-h before exercise had no impact on the recovery of lower limb muscular endurance (one set at $100 \%$ of $10 \mathrm{RM}$ leg press and hack squat), markers of muscle damage (creatine kinase), or electromyographic (EMG) activation compared to a placebo at 24,48 and $72 \mathrm{~h}$ post-exercise. These author's findings support earlier similar work displaying negligible changes in protein signaling and synthesis rates in elderly males, although this was with supplementation of L-citrulline (Churchward-Venne et al. 2014). It could be argued, however, that the exercise selected in da Silva et al. (2017) was not damaging enough to appropriately assess the recovery of muscle function. Specifically, between 24 and $72 \mathrm{~h}$ post-exercise the repetitions completed were similar that suggests the participants were not sufficiently fatigued at the 24-h time point. It is suggested a reduction of between 15-60\% from baseline performance, which can persist for up to 2 weeks, is required to suggest exercise-induced muscle damage (Owens et al. 2019). In support, the creatine kinase concentrations were low $\left(\sim 350 \mathrm{UL}^{-1}\right)$, which compared to other literature, values greater than $1000 \mathrm{UL}^{-1}$ are typically seen (Inman et al. 2018; Ehlers et al. 2002). Lastly, the crossover trial study design employed by da Silva et al. (2017) is arguably not the most appropriate to assess recovery from exercise-induced muscle damage; instead, a matched groups design could have been used to mitigate the repeated bout effect, particularly as da Silva et al. (2017) used recreational participants. As a result, based on the limited research to date and methodological limitations, further research is warranted.

\section{Conclusions and future directions}

The lack of positive effects from CM supplementation within the existing literature is due to a number of factors, including the testing protocols not featuring a predominantly aerobic energy contribution, the lack of test-retest reliability of exercise protocols, dosing strategy (i.e., amount and timing), and the recent discovery of quality control issues with some manufacturers stated citrulline:malate ratios. Indeed, this diversity adds a level of additional noise to our ability to draw firm conclusions about the efficacy of CM supplementation on exercise performance or recovery from exhaustive exercise. Nevertheless, from the available evidence, an acute $8 \mathrm{~g}$ dose CM may, albeit not consistently, increase muscular endurance-strength performance (Table 1). This corroborates with a recent meta-analysis conducted at the time of writing this review, which also reported similar benefits (Vårvik et al. 2021). Whereas, there is little evidence to advocate its use in the production and maintenance of muscular power, maximal strength, recovery of muscular function, or supporting muscular adaptations currently (Table 1). Lastly, athletes wishing to explore NO enhancers are reminded that a good level of evidence exists for L-citrulline to improve exercise performance, and therefore may consider use of this supplement whilst the intricacies of CM supplementation are discovered (Gonzalez and Trexler 2020).

Future research should investigate the bioavailability of key variables, namely plasma NO, citrulline, and arginine following a range of doses of CM. Only one study exists that has reported this important data at the time of writing this review, and this did not include the malate component. 
Only at this point will the physiologically optimal dose of $\mathrm{CM}$ become clear. Due to the logistical and cost burden of conducting such a study, a simpler approach would be to assess various doses of $\mathrm{CM}$ (e.g., 8 vs. 10 vs. $12 \mathrm{~g} \mathrm{CM}$ ) on an exercise protocol that requires a predominantly aerobic energy demand and has high test-retest reliability. Furthermore, manufacturers are required to take more responsibility to guarantee that the ratio stated is what is contained within the product, and researchers/practitioners should be aware of this when sourcing their product for research and/or use with athletes. Finally, those who have the resources (primarily manufacturers but also researchers) should analyse the purity of the C:M ratio to ensure they have every opportunity of achieving an ergogenic effect.

Author contributions LG conceived and designed the research. LG wrote the manuscript, with contributions from CAB and SAS. All other authors offered critical comments and approved the manuscript.

\section{Declarations}

Conflict of interest The authors declare no conflict of interest.

Open Access This article is licensed under a Creative Commons Attribution 4.0 International License, which permits use, sharing, adaptation, distribution and reproduction in any medium or format, as long as you give appropriate credit to the original author(s) and the source, provide a link to the Creative Commons licence, and indicate if changes were made. The images or other third party material in this article are included in the article's Creative Commons licence, unless indicated otherwise in a credit line to the material. If material is not included in the article's Creative Commons licence and your intended use is not permitted by statutory regulation or exceeds the permitted use, you will need to obtain permission directly from the copyright holder. To view a copy of this licence, visit http://creativecommons.org/licenses/by/4.0/.

\section{References}

Agudelo LZ, Ferreira DM, Dadvar S, Cervenka I, Ketscher L, Izadi M, Ruas JL (2019) Skeletal muscle PGC- $1 \alpha 1$ reroutes kynurenine metabolism to increase energy efficiency and fatigue-resistance. Nat Commun 10(1):1-12

Bahri S, Curis E, El Wafi FZ, Aussel C, Chaumeil JC, Cynober L, Zerrouk N (2008) Mechanisms and kinetics of citrulline uptake in a model of human intestinal epithelial cells. Clin Nutr 27(6):872-880

Bendahan D, Mattei JP, Ghattas B, Confort-Gouny S, Le Guern ME, Cozzone PJ (2002) Citrulline/malate promotes aerobic energy production in human exercising muscle. Br J Sports Med 36(4):282-289

Breuillard C, Cynober L, Moinard C (2015) Citrulline and nitrogen homeostasis: an overview. Amino Acids 47(4):685-691

Casonatto J, Enokida DM, Grandolfi K (2019) Inter-Individual Responses to Citrulline Malate Oral Supplementation on PostExercise Hypotension in Hypertensives: A 24-Hour Analysis. Arq Bras Cardiol 113(2):218-228
Chappell AJ, Allwood DM, Simper TN (2018a) Citrulline malate fails to improve german volume training performance in healthy young men and women. J Diet Suppl 21:1-12

Chappell AJ, Allwood DM, Johns R, Brown S, Sultana K, Anand A et al (2018b) Citrulline malate supplementation does not improve german volume training performance or reduce muscle soreness in moderately trained males and females. J Int Soc Sports Nutr 15(1):8

Churchward-Venne TA, Cotie LM, MacDonald MJ, Mitchell CJ, Prior T, Baker SK et al (2014) Citrulline does not enhance blood flow, microvascular circulation, or myofibrillar protein synthesis in elderly men at rest or following exercise. Am J Physiol 307:83

Cunniffe B, Papageorgiou M, O'Brien B, Davies NA, Grimble GK, Cardinale M (2016) Acute citrulline-malate supplementation and high-intensity cycling performance. J Strength Cond Res 30(9):2638-2647

Currell K, Jeukendrup AE (2008) Validity, reliability and sensitivity of measures of sporting performance. Sports Med 38:297-316

da Silva DK, Jacinto JL, de Andrade WB, Roveratti MC, Estoche JM, Balvedi MCW et al (2017) Citrulline malate does not improve muscle recovery after resistance exercise in untrained young adult men. Nutrients 9(10):1132

Davis AR, Webber CL, Fish WW, Wehner TC, King S, Perkins-Veazie $P$ (2011) L-citrulline levels in watermelon cultigens tested in two environments. HortScience 46(12):1572-1575

Ehlers GG, Ball TE, Liston L (2002) Creatine kinase levels are elevated during 2-a-day practices in collegiate football players. J Athl Train 37(2): 151

Farney TM, Bliss MV, Hearon CM, Salazar DA (2019) The effect of citrulline malate supplementation on muscle fatigue among healthy participants. J Strength Cond Res 33(9):2464-2470

Gibala MJ, Young ME, Taegtmeyer H (2000) Anaplerosis of the citric acid cycle: role in energy metabolism of heart and skeletal muscle. Acta Physiol Scand 168(4):657-665

Gills JL, Glenn JM, Gray M, Romer B, Lu H (2021) Acute citrullinemalate supplementation is ineffective during aerobic cycling and subsequent anaerobic performance in recreationally active males. Eur J Sport Sci 18:1-7

Glenn JM, Gray M, Wethington LN, Stone MS, Stewart RW, Moyen NE (2017) Acute citrulline malate supplementation improves upper- and lower-body submaximal weightlifting exercise performance in resistance-trained females. Eur J Nutr 56(2):775-784

Gonzalez AM, Trexler ET (2020) Effects of citrulline supplementation on exercise performance in humans: A review of the current literature. J Strength Cond Res 34(5):1480-1495

Gonzalez AM, Spitz RW, Ghigiarelli JJ, Sell KM, Mangine GT (2018) Acute effect of citrulline malate supplementation on upper-body resistance exercise performance in recreationally resistancetrained men. J Strength Cond Res 32(11):3088-3094

Grimble GK (2007) Adverse gastrointestinal effects of arginine and related amino acids. J Nutr 6(2):1693-1701

Hargreaves M, Spriet LL (2020) Skeletal muscle energy metabolism during exercise. Nat Metab 2:817-828

Hultström M, Amorim de Paula C, Fontes AP, M., Porcelli, S., Bellistri, G., Pugliese, L., et al (2015) Commentaries on Viewpoint: Can elite athletes benefit from dietary nitrate supplementation? J Appl Physiol 119(6):762-769

Hwang P, Marroquín FEM, Gann J, Andre T, McKinley-Barnard S, Kim C et al (2018a) Eight weeks of resistance training in conjunction with glutathione and L-Citrulline supplementation increases lean mass and has no adverse effects on blood clinical safety markers in resistance-trained males. J Int Soc Sports Nutr 15(1):1-10

Hwang P, Morales Marroquin FE, Gann J, Andre T, McKinley-Barnard S, Kim C et al (2018b) Eight weeks of resistance training in conjunction with glutathione and L-citrulline supplementation increases lean mass and has no adverse effects on blood clinical 
safety markers in resistance-trained males. J Int Soc Sports Nutr 15(1):30

Inman LA, Rennie MJ, Watsford ML, Gibbs NJ, Green J et al (2018) Reference values for the creatine kinase response to professional Australian football match-play. J Sci Med Sport 21(8):852-857

Jones AM, Vanhatalo A, Seals DR, Rossman MJ, Piknova B, Jonvik KL (2020) Dietary nitrate and nitric oxide metabolism: mouth, circulation, skeletal muscle, and exercise performance. Med Sci Sports Exerc 53(2):280-294

Lucero AA, Addae G, Lawrence W, Neway B, Credeur DP, Faulkner J, Stoner L (2018) Reliability of muscle blood flow and oxygen consumption response from exercise using near-infrared spectroscopy. Exp Physiol 103(1):90-100

Moinard C, Nicolis I, Neveux N, Darquy S, Benazeth S, Cynober L (2008) Dose-ranging effects of citrulline administration on plasma amino acids and hormonal patterns in healthy subjects: The citrudose pharmacokinetic study. Br J Nutr 99(4):855-862

Owens DJ, Twist C, Cobley JN, Howatson G, Close GL (2019) Exercise-induced muscle damage: What is it, what causes it and what are the nutritional solutions? Eur J Sport Sci 19(1):71-85

Perez-Guisado J, Jakeman PM (2010) Citrulline malate enhances athletic anaerobic performance and relieves muscle soreness. J Strength Cond Res 24(5):1215-1222

Schwedhelm E, Maas R, Freese R, Jung D, Lukacs Z, Jambrecina A et al (2008) Pharmacokinetic and pharmacodynamic properties of oral L-citrulline and L-arginine: impact on nitric oxide metabolism. Br J Clin Pharmacol 65(1):51-59

Sindelar JJ, Milkowski AL (2012) Human safety controversies surrounding nitrate and nitrite in the diet. Nitric Oxide 26(4):259-266

Stamler JS, Meissner G (2001) Physiology of nitric oxide in skeletal muscle. Physiol Rev 81(1):209-237

Takeda K, Machida M, Kohara A, Omi N, Takemasa T (2011) Effects of citrulline supplementation on fatigue and exercise performance in mice. J Nutr Sci Vitaminol 57(3):246-250
Trexler ET, Persky AM, Ryan ED, Schwartz TA, Stoner L, Smith-Ryan AE (2019a) Acute effects of citrulline supplementation on highintensity strength and power performance: a systematic review and meta-analysis. Sports Med 49(5):707-718

Trexler ET, Keith DS, Schwartz TA, Ryan ED, Stoner L, Persky AM, Smith-Ryan AE (2019b) Effects of citrulline malate and beetroot juice supplementation on blood flow, energy metabolism, and performance during maximum effort leg extension exercise. The J Strength Cond Res 33(9):2321-2329

Vanhoutte PM, Zhao Y, Xu A, Leung SW (2016) Thirty years of saying NO: sources, fate, actions, and misfortunes of the endotheliumderived vasodilator mediator. Circ Res 119(2):375-396

Vårvik FT, Bjørnsen T, Gonzalez AM (2021) Acute effect of citrulline malate on repetition performance during strength training: a systematic review and meta-analysis. Int J Sport Nutrit Exer Metabol 1(aop):1-9

Wax B, Kavazis AN, Weldon K, Sperlak J (2015) Effects of supplemental citrulline malate ingestion during repeated bouts of lower-body exercise in advanced weightlifters. J Strength Cond Res 29(3):786-792

Wax B, Kavazis AN, Luckett W (2016) Effects of supplemental citrulline-malate ingestion on blood lactate, cardiovascular dynamics, and resistance exercise performance in trained males. J Diet Suppl 13(3):269-282

Wu JL, Wu QP, Huang JM, Chen R, Cai M, Tan JB (2007) Effects of L-malate on physical stamina and activities of enzymes related to the malate-aspartate shuttle in liver of mice. Physiol Res 56(2):213-220

Publisher's Note Springer Nature remains neutral with regard to jurisdictional claims in published maps and institutional affiliations. 